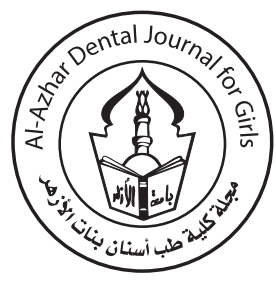

\title{
Evaluation of the Antimicrobial Effect of Pomegranate Extract on Streptococcus Mutans
}

\author{
Manal S. El-Sharkawy ${ }^{1^{*}}$, Mohamed H. Mostafa ${ }^{2}$, Magda A. El- Malt ${ }^{3}$
}

Codex : $57 / 1910$

azhardentj@azhar.edu.eg

http://adjg.journals.ekb.eg

DOI: $10.21608 /$ adjg.2019.7614.1084

\begin{abstract}
Purpose: This study was conducted to evaluate the effect of two mouth rinses (pomegranate fresh juice and pomegranate peel extract), against chlorhexidine mouthwash on salivary S.mutans count in a group of Egyptian children. Material and Methods: A total of 45 Egyptian children from both sex were included in this study. Children age ranging from 5 to 10 years old in a good physical condition. Children randomly were distributed into three Groups A, B \& C, each of 15 children in each group. Group A (using pomegranate fresh juice): consisted of fifteen children, each participant was instructed to rinse with $35 \mathrm{ml}$ of pomegranate fresh juice wash about 2 minutes. Group B (using pomegranate peel extract): consisted of fifteen children, each participant was instructed to rinse with $35 \mathrm{ml}$ of pomegranate peel extract wash about 2 minutes. Group C (Control group chlorhexidine mouthwash $0.2 \%$ ): consisted of fifteen children, each participant was instructed to rinse with $35 \mathrm{ml}$ of chlorhexidine mouthwash wash about 2 minutes. Results: There was a significant reduction in mean Streptococcus mutans count in the 3 groups after the study. Conclusions: Pomegranate (peel \& juice) mouthwash was successful as an antimicrobial agents. It significantly reduced the total bacterial count in the saliva of children when compared by a potent antiseptic like $0.2 \%$ chlorhexidine.
\end{abstract}

\section{INTRODUCTION}

Dental caries is one of the most prevalent oral disease in the world. It affects a lot of people with different age groups during their lifetime, it is notice as an infectious disease lead to effective and changes the oral environment, which then results the hard tooth tissues has localized destruction. Dental caries is a disease occurs when hard tissues was softened by demineralization of the tooth caused by sugars and bacteria on foods, then producing an acid that made hard tooth

Pomegranate,

Chlorhexidine mouthwash, oral microorganisms.

- Paper extracted from master thesis titled "Evaluation of the Antimicrobial Effect of Pomegranate Extract on Streptococcus Mutans"

1. * Post graduate student, Department of Pedodontics and Oral Health, Faculty of Dental Medicine for Girls, Al-Azhar University, Egypt. Email: doctormsf90@gmail.com.

2. Assistant Professor, Head of Pedodontics and oral Health Department, Faculty of Dental Medicine for Girls, Al-Azhar University, Egypt.

3. Assistant Professor, Pedodontics and oral Health Department, Faculty of Dental Medicine for Girls, Al-Azhar University, Egypt. 
structure demineralizes ${ }^{(1)}$. The development of the carious lesion is correlating with infection, chewing difficulty, pain, early teeth loss and trauma.

Dental pain is the instantaneously most common effect of untreated tooth caries, if the children have dental pain their effected on school performance can be impaired as well their daily activities influenced as playing, sleeping and eating ${ }^{(2)}$. Streptococcus mutans is the common bacteria occurred in the mouth cavity it is a facultative anaerobic bacterium grampositive cocci and it is a considerable contributor roles to tooth decay. The central role of dental caries etiology Streptococcus sobrinus and Streptococcus mutans that has been adhered to plaque bacteria and to the salivary pellicle also this acidogenic bacteria were able when presence of sucrose to form polysaccharides ${ }^{(3)}$. Punica granatum documented as the pomegranate is a shrub native from Asia.

It is one of the oldest fruit which has not changed much throughout the history of humaneness. It has been excessively consumed in conventional medicine in the world for the curing of various kinds of disease $^{(4)}$. Pomegranates (having antibacterial properties) suggested from various studies which are generally treated as real antibiotics and the most important avail is that antibiotic-resistant pathogens will be easily killed by these natural antimicrobials as they are available for no cost and no have adverse effects ${ }^{(5)}$.

Pomegranates are powerful antioxidants because contain tannins and polyphenols. The antioxidant activity contributed to red fruit's anthocyanidins (red pigment) therefore pomegranate juice has more bioactivity compared to its purified polyphenols ${ }^{(6)}$. In the worldwide the pomegranate one of the most natural fruits consumed because its pleasant taste, medicinal properties and high nutritional values; it is used in the treatment of ulcers, aphthae (mouth ulcers), diarrhea, dysentery, parasitic infections, diabetes and cardiovascular disease ${ }^{(7)}$.

\section{MATERIAL AND METHODS}

Materials used: Pomegranate identified as punica granatum were collected from a local market, Cairo city, Egypt, two kinds of the pomegranate extract were prepared Pomegranate fresh juice and Pomegranate peel extract. Chlorhexidine mouthwash $(0.2 \%)$ : (Oraxine, medical cosmetic products Ltd, KSA) and Mitis Salivarius Bacitracin (MSB): Base enriched with sucrose to selectively isolated oral streptococci and inhibits other micro-organisms.

Case Selection: 45 children were visited the department of Paedodontics, Ethical approval was obtained from the Research and Ethics committee, Dental College, Al-Azhar University (Girl's branch). Inclusion criteria: cooperative children age ranging from 5 to 10 years old with a good physical condition, No history of recent antibiotic administration (previous week) or anti-microbial mouth rinse (previous 12 hours). Exclusion criteria: children with systemic disease, children using any other oral hygiene aid other than routine teeth brushing, children with history of recent antibiotic administration or antimicrobial mouth rinse, or topical fluoride treatment, children with known history of allergy to any mouthwash or drug.

Methods: Children randomly were distributed into 3 Groups A, B \& C, 15 children in each group. (Group A) consisted of 15 children using pomegranate fresh juice mouth wash was prepared by separating pomegranate arils from the fruits and then mixed in the electric mixer and then filtered from the pomegranate seeds. (Group B) consisted of 15 children using pomegranate peel extract mouthwash was prepared by drying the pomegranate peels and grinding them into the fine powder, for each rinsing $2 \mathrm{~g}$ of pomegranate peel dissolve in $35 \mathrm{ml}$ of distilled water at $100{ }^{\circ} \mathrm{C}$ for 3 minutes. Several layers of gauze were used to refinery the material. (Group C) consisted of 15 children using chlorhexidine mouthwash $(0.2 \%)$. Instructed to 
each subject to rinse with $35 \mathrm{ml}$ of mouthwash wash 2 minutes, in the morning before breakfast or at least 1 hour after breakfast. Two salivary sample were collected from each child immediately and after 60 minutes of using the mouth wash.

\section{Collection of saliva sample}

Unstimulated saliva was taken from each child by asking him to spit in a sterile plastic container until a suitable amounts of saliva was collected (in the morning before breakfast or at least 1 hour after breakfast).

Baseline sample (S1): The initial sample was taken before using the mouth wash by asking the child to spit in a sterile plastic container.

Second sample (S2): The second sample was taken after 60 minutes for using specific mouth wash by asking the child to spit in a sterile plastic container (Table 1).

Table(1): Two saliva samples (S1\&S2) are taken for each individual as shown in the following table:

\begin{tabular}{|c|l|}
\hline Sample & \multicolumn{1}{|c|}{ Description } \\
\hline S1 & Initial sample \\
\hline S2 & After 60 minutes of using mouth wash \\
\hline
\end{tabular}

\section{Preparation of the media}

The selective medium Mitis salivarius bacitracin was prepared as following:

1. 1 liter of distilled water used to dissolve 90 grams of dehydrated mitis salivarius agar.

2. The medium was heated to components dissolved and then at $121^{\circ} \mathrm{C}$ autoclaved for fifteen minutes .

3. The medium was left to cool to $55^{\circ} \mathrm{C}$ then $1 \mathrm{ml}$ of $1 \%$ sterilized potassium tellurite and $1 \mathrm{ml}$ of 200 units $1 \mathrm{ml}$ sterilized bacitracin was added.
Sterilization of tellurite was done by filtration.

4. Each plate was poured with approximately 20 $\mathrm{ml}$ of the medium and then to dry $24 \mathrm{hrs}$ at room temperature under $5-10 \% \mathrm{CO} 2$ tension (incubator).

5. Plates in the refrigerator were stocked at $4^{\circ} \mathrm{C}$ unit use ${ }^{(8)}$

\section{Microbial Analysis}

1. The saliva samples were diluted at least four dilutions $1.10^{-1}, 1.10^{-2}, 1.10^{-3}, 1.10^{-4}$ using sterile diluted (peptone water).

2. 0.1 of each dilution was plated on the surface of selective media used (mitis salivarius agar). Each sample was cultured in triplicate.

3 . The plates were incubated anaerobically in the candle jar at $37^{\circ} \mathrm{C}$ for $2-3$ days.

4. Streptococcus mutans colonies were examined systematically and the number of the colony units (CFU / $\mathrm{ml}$ ) of the original saliva selected.

\section{Statistical Analysis}

Statistical analysis was then performed using a commercially available software program (SPSS 19; SPSS, Chicago, IL, USA) to compare the mean bacterial count within and between groups.

As data was parametric, the significance of the difference between each group was predestined using one way analysis of variance (ANOVA) test and Tukey's post hoc test.

Pre- treatment and post treatment mean values were compared using paired t-test.

The percentage of change was calculated by the following formula:

Value after-value beforeX100

Value before the level of significance was set at $\mathrm{P}<0.05$. 
Table (2): Comparison of mean percent change in colony forming unit of Streptococcus mutans (\%) in Pomegranate fresh juice, Pomegranate peel extract and chlorohexidine groups after treatment

\begin{tabular}{|c|c|c|c|c|c|c|c|c|c|}
\hline \multirow[t]{2}{*}{ Groups } & \multirow[t]{2}{*}{ Mean } & \multirow{2}{*}{$\begin{array}{l}\text { Std. } \\
\text { Dev }\end{array}$} & \multirow{2}{*}{$\begin{array}{l}\text { Std. } \\
\text { Error }\end{array}$} & \multicolumn{2}{|c|}{$\begin{array}{l}\text { 95\% Confidence } \\
\text { Interval for Mean }\end{array}$} & \multirow[t]{2}{*}{ Min } & \multirow[t]{2}{*}{ Max } & \multirow[t]{2}{*}{$\mathbf{F}$} & \multirow[t]{2}{*}{$\mathbf{P}$} \\
\hline & & & & Lower Bound & Upper Bound & & & & \\
\hline $\begin{array}{c}\text { Pomegranate } \\
\text { fresh juice }\end{array}$ & $-99.75^{a}$ & .26 & .04 & -99.83 & -99.68 & -99.98 & -98.94 & 32.8 & $.000 *$ \\
\hline $\begin{array}{c}\text { Pomegranate } \\
\text { peel extract }\end{array}$ & $-100.0^{\mathrm{b}}$ & .00 & .00 & -100.00 & -100.00 & -100 & -99.97 & & \\
\hline Chlorohexidine & $-99.96^{b}$ & .07 & .01 & -99.98 & -99.94 & -100 & -99.72 & & \\
\hline
\end{tabular}

Significance level $p<0.05$, *significant, $n s=$ non-significant

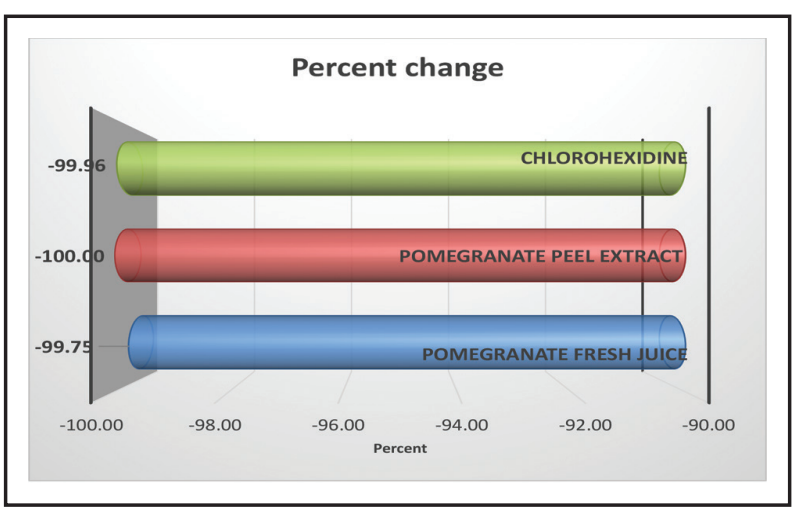

Fig. (1) Bar chart showing mean percent change in colony forming unit of Streptococcus mutans (\%) in Pomegranate fresh juice, Pomegranate peel extract and chlorohexidine groups after treatment

\section{RESULTS}

The results of the current study (Table 2) \& (Figure 1) showed that rinsing with pomegranate peel extract mouthwash had a statistically significant more amount reduction of S.mutans count (CFU) (100\%), followed by chlorhexidine (99.96\%) and finally pomegranate fresh juice $(99.75 \%)$ had the least reduction of S.mutans count $(\mathrm{CFU})$.

\section{DISCUSSION}

Dental caries is a chronic infectious oral disease has most common affected in teenagers and children in the worldwide; this infectious disease without early prevention can lead to destroyed of hard dental tissue and lately may tooth loss ${ }^{(9)}$.
Mutans streptococci are groups of cariogenic bacteria properties and their potency to yield intracellular and extracellular polysaccharides that expedite bacterial attachment on surface of the tooth. Streptococcus mutans had been embroiled as the principle bacterial component responsible for progression of dental caries. It is the standard microorganism related to coronal caries, and was found to be among the first microorganisms to colonize infants shortly after teeth eruption ${ }^{(10)}$.

In the present study pomegranate fruits were collected from the local market, Cairo, Egypt. The study to measure the levels of Streptococcus mutans by saliva is one of the most popular methods for recognize subjects at risk of dental caries. There is a close correlation between the levels of Streptococcus mutans in saliva and its levels in plaque ${ }^{(11)}$. In this study saliva samples were preferred because it is easier and it reflects accurately the caries experience and risk in every individual, this was in accordance with different studies which assessed the salivary Streptococcus mutans level in different patient categories as a judge for the effectiveness of different mouthwashes on patient caries risk ${ }^{(12-}$ 15). Mitis Salivarius Bactitracin (MSB) media was selected for detection and counting of colonies of Streptococcus mutans because it is selective for such colonies. The addition of bacitracin to the media allows Streptococcus mutans to grow and form colonies and inhibit the growth of most other oral bacteria. Despite MSB shortcomings, it remains 
a reference method for detection of Streptococcus mutans isolation ${ }^{(11,16)}$. Chlorhexidine has been supposed as a "gold" standard for the inhibition of plaque, gingivitis and have antibacterial properties. However, it presents local adverse effects when used for prolonged periods such as brown discoloration of teeth, restorative materials and dorsum of the tongue, oral mucosal ulceration, taste trouble, ulcer in oral mucosa, unilateral/bilateral parotid gland swelling and enhanced supragingival calculus formation on its long term use ${ }^{(17,18)}$.

The present study was conducted to evaluate the effect of two mouthwash, pomegranate fresh juice and pomegranate peel extract. The distilled water was used for preparation of pomegranate mouthwash, against chlorhexidine mouthwash on salivary Streptococcus mutans count in Egyptian children groups. The distilled water when used for preparation of the mouthwash is better than alcohol used in oral care products because it can be irritating to the cheeks, teeth and gum. Generally, excessive use of products that contain alcohol may also weaken the immune system natural ability to fight bacteria and illness ${ }^{(19)}$. Also this method is safe, economical, easy to prepare and can be used as a home care product.

In the current study, the age of selected children ranged from 5 to 10 years, the children were instructed used mouthwash and to rinse for 2 minutes and were collected the saliva samples from the children, before using the mouthwash and after 60 minutes of use. This age was chosen as they can easily rinse their mouth without swallowing the mouthwash, this was in agreement with result of another study applied on children with age ranged similar to our study range ${ }^{(13)}$. The time used for rinsing agreed with another studies, who were collected saliva also samples before rinsing and after 60 minutes from mouthrinsing ${ }^{(13,14)}$.

The results of the current study showed that rinsing with pomegranate peel extract mouthwash had a statistically significant more reduction of Streptococcus mutans count (CFU) (100\%), followed by chlorhexidine (99.96\%) and finally pomegranate fresh juice $(99.75 \%)$ had the least reduction of Streptococcus mutans count(CFU).

These results were in agreement with a similar an in-vivo study demonstrated the effect of rinsing with pomegranate peel extract in comparison with chlorhexidine mouth-rinse $(0.2 \%)$ on streptococcus mutans count, using saliva samples before and after rinsing of the patients. This study showed using peel extract mouth-rinse significantly decrease in Streptococcus mutans count more than chlorhexidine ${ }^{(13,14)}$. This study reveal that, pomegranate peel extract mouthrinse possesses antimicrobial activity against Streptococcus mutans occure in the oral cavity of children as in vivo study, and may be used as an adjunct to prevent dental caries and keep good oral hygiene in children (13). This result came in coordinate with an in-vivo study which evaluated the effect of pomegranate peel extract and camellia sinesis mouthwashes in comparison with chlorhexidine mouthwash on the level of plaque and gingival index. The study observed that the group used punica granatum significantly improved the plaque and gingival status at all sites ${ }^{(20)}$.

The results of the our study, however, came in contrary with an in vitro study which evaluated antimicrobial effect of hydroalcoholic extracts of pomegranate juice and peel against two different strains of Streptococcus mutans, then evaluated the values of minimum bactericidal concentration and minimum inhibitory concentration were determined against the two different strains of Streptococcus mutans. The results showed the pomegranate juice extract had higher inhibitory activity against the two strains than peel extract. They concluded that microbiological tests demonstrated the extracts of pomegranate peel and juice are capable to change mainly cariogenic bacteria and tooth decay ${ }^{(21)}$. 
Although the result of present study showed that rinsing with pomegranate fresh juice mouthwash caused the least reduction in percentage $(99.75 \%)$ on Streptococcus mutans count when compared with other groups however, the result showed that it had high antibacterial effect, This agreed with an in vivo study which demonstrated the effect of rinsing with hydroalcoholic extract from pomegranate fruit as an antigingivitis and antiplaque in compared with chlorhexidine. Also their results showed improvement in bleeding and gingivitis score more than chlorhexidine ${ }^{(22)}$. The results of our study agreed also with another study evaluated antibacterial effect of pomegranate juice on dental plaque microorganisms. There finding showed the mean number of CFU on lactobacilli and Streptococcus mutans before and after rinsing pomegranate juice and the percentage of reduction in the number of $\mathrm{CFU}$ was $32 \%$. There was greater reduction in the lactobacilli strains (46\%) compared to streptococci strains $(23 \%)^{(6)}$.

The results of the present study, however, came in obverse with an in vivo study which evaluated antimicrobial activity of chlorhexidine, distilled water and pomegranate juice mouthrinse during wearing orthodontic appliances. The results showed pomegranate juice is very effective to reduce dental plaque compared to chlorhexidine and distilled water in fixed orthodontic patients. Pomegranate juice is tasty, easily prepared and has mild antimicrobial potency which is beneficial for long-term use. Preferable during orthodontic treatment is healthy periodontal tissue, pomegranate juice salutary effect and safety if mouthrinse daily use in orthodontic patients, it can be suggested as an ideal long term use mouthwash for fixed orthodontic patients ${ }^{(23)}$. And agreed with other in vitro study evaluated the effect of pomegranate extract on bacterial biofilm and five oral bacteria formation on orthodontic wire, the results showed antibacterial properties of punica granatum and prevented orthodontic wire bacterial biofilm formation ${ }^{(24)}$.
The results of the current study were in agreement with an in vivo study evaluated the effect of the hydroalcoholic extract for pomegranate fruits on dental plaque microorganisms when compared with distilled water, chlorhexidine. The results showed the pomegranate was very efficacious against dental plaque microorganisms decreasing the $\mathrm{CFU} / \mathrm{ml}$ $(84 \%)$ followed by chlorhexidine $(79 \%)$ and the least inhibition was demonstrated in the distilled water group only an (11\%) inhibition of $\mathrm{CFU} / \mathrm{ml}{ }^{(25)}$. This result came in coordinate with an in-vitro study which evaluated the efficacious of pomegranate peels extract against Streptococcus mutans and the adhesion to surface of the teeth in comparison with chlorhexidine gluconate. The result demonstrated the inhibition zones diameters of Streptococcus mutans was higher to pomegranate peels extract than chlorhexidine ${ }^{(26)}$.

\section{CONCLUSIONS}

Pomegranate (peel \& juice) mouthwash was successful as an antimicrobial agents. It significantly reduced the total bacterial count in the saliva of children when compared by a potent antiseptic like $0.2 \%$ chlorhexidine.

\section{REFERENCES}

1. Selwitz RH, Ismail AI, Pitts NB. Dental caries. Lancet 2007; 369, 51-9.

2. Edelstein BL, Ureles SD, Smaldone A. Very high salivary streptococcus mutans predicts caries progression in young children. Pediatr Dent 2016; 38, 325-30.

3. Marsh, P.D. Are dental diseases examples of ecological catastrophes. Microbiology-Sgm. 2003, 149, 279-94.

4. Dahham SS, Ali MN, Tabassum H, Khan M. Studies on antibacterial and antifungal activity of pomegranate (Punica granatum L.). American-Eurasian J Agric \& Environ Sci 2010; 9, 273-81.

5. Ramesh KS, Shamim SF. Role of pomegranate in preventive dentistry. IJRAP 2012; 3, 5-10.

6. Sowmya K, Sunder K, Lakshminarayan N, Effect of Pomegranate Juice on Dental Plaque Microorganisms Streptococci and Lactobacilli, Anc Sci Life. 2011;31: 49-51. 
7. Dell'Agli, Taramelli, Lucantoni L., Habluetzel, Bhattacharya, Bosisio, et al. Antiplasmodial activity of Punica granatum L. fruit rind. J. Ethnopharmacol, 2009; 125, 279-85

8. Petti S, Pezzit S, Cattaruzza JF, Area AS. Restoration related salivary streptococcus mutans level: a dental caries risk factor. J Dent.1997; 25:257- 62.

9. Nurelhuda NM, Al-Haroni M, Trovik TA et al. Caries experience and quantification of Streptococcus mutans and Streptococcus sobrinus in saliva of Sudanese schoolchildren. Caries Res 2010; 44, 402-7.

10. Fan C, Wang W, Xu T et al. Risk factors of early childhood caries among children in Beijing: a case-control study. BMC Oral Health 2016; 16: 98.

11. Baca P, Castillo AM, Baca AP, liebana MJ, Junco P, Liebana J. Gentotypes of streptococcus mutans in saliva versus dental plaque. Archives of oral Biology J. 2008; 53: 751-4.

12. Robert A.D, Daniel J.D and David J.D. Pomegranate Extract Mouth Rinsing Effects on Saliva Measures Relevant to Gingivitis Risk. Phytotherapy Research Phytother. 2009; 23: 1123-7.

13. Sham S.B, Sundeep H.K, Mohammed F. Effect of pomegranate extract mouthrinse on salivary $\mathrm{PH}$ and Streptococcus mutans counts in children an in vitro study, 2014; 4, 12-9.

14. Dilshad U, Bahija D, Mohammed F, Arshiya A, and Kusai B. The effect of pomegranate mouthrinse on Streptococcus mutans count and salivary $\mathrm{pH}$ : An in vivo study. J Adv Pharm Technol Res. 2016; 7: 13-6.

15. Sari and Birinci I. Microbiological evaluation of $0.2 \%$ Chlorhexidine gluconate mouth rinse in orthodontic patients. Angle Orthodont.2007; 77:881- 4.

16. Murray JJ, June HN, and Fames GS. Fluoride and dental Caries In: Prevention of oral disease. Ed4, Oxford University Press, 2003;49-59.

17. Kaur H, Jain S, Kaur A. Comparative evaluation of the anti-plaque effectiveness of green tea catechin mouthwash with chlorhexidine gluconate. J Indian Soc Periodontol. $2014 ; 18: 178-82$

18. Mali AM, Behal R, Gilda SS. Comparative evaluation of $0.1 \%$ turmeric mouthwash with $0.2 \%$ chlorhexidine gluconate in prevention of plaque and gingivitis: A clinical and microbiological study. J Int Soc Prev Community Dent. 2012;16:386-91

19. Romeo J, Warnberg J, Nova E, Diaz LE, Gomez-Martinez S, Marcos A. Moderate alcohol consumption and the immunesystem: a review. Br J Nutr. 2007;98:111-5.

20. Harshal RC, Nirma Y, Barkha M, Neema S, Aditi M, Balaji M. Effectiveness of Indigenously Prepared Punica Granatum and Camellia Sinesis Mouthwashes as an adjunct to Non Surgical Periodontal Therapy. J Nep Soc Perio Oral Implantol. 2017;1:27-31.

21. Gianmaria FF, Elisa S, Daniela S, Gabiria P, Roberta C, Chiara P, et al. In Vitro Antibacterial Activity of Pomegranate Juice and Peel Extracts on Cariogenic Bacteria. Biomed Res Int.2017; 5: 1-7.

22. Sakshi A, Vidya D, Bhavna JK, Praful M, Pankaj K, A comparative evaluation of efficacy of Punica granatum and chlorhexidine on plaque and gingivitis. J Int Clin Dent Res Organ. 2011; 3, 29-32.

23. Haryono U and Kimberly CO, Pomegranate juice (Punica granatum) as an ideal mouthrinse for fixed orthodontic patients Dent. J. (Maj. Ked. Gigi), 2012; 45 , 221-7.

24. Zahra A, Elahe VD, Marzieh G, Parisa A, Mohammad K, Arash M. Effect of Punica granatum L. Flower Water Extract on Five Common Oral Bacteria and Bacterial Biofilm Formation on Orthodontic Wire. Iran J Public Health. 2014;43: 1688-94.

25. Silvana MS, Menezes, Luciana NC, Glauce SB. Punica granatum (Pomegranate) Extract Is Active Against Dental Plaque. J Herb Pharmacother. 2009; 20: 79-92.

26. Zainab AA, Maha AM, Ghada IT, Rasha MS. The effect of Pomegranate Peels Aqueous Extract against Streptococcus Mutans and the Adherence to tooth surface in Comparison to Chlorhexidine Gluconate (in Vitro Study). IISTE Journal. 2015;.35: 28-32. 